\title{
The Common Features of The Geometry Angle Applied for Hard Cutting Materials
}

\author{
Z.Y.Wu, X.Y. He, K.W.Ji, X.J.Tang \\ National Key Laboratory for Equipment \\ Remanufacturing, Academy of Armored Force \\ Engineering ,Beijing 100072, China \\ e-mail:Wu_zhiyuan20021@163.com
}

\author{
X.Y. He \\ Shijiazhuang Mechanized Infantry Academy, \\ Shijiazhuang 050000, China
}

\begin{abstract}
With the continuous development of science and technology, the high hardness materials are finding wider and wider application in all fields. But the high hardness make it hard to cut them, which has been a bottleneck to their further application. In this paper, about three hundreds papers on hardness materials cutting were compared, and the distribution of optimal geometry angle was summarized and analyzed. The results can be useful to the related researches.
\end{abstract}

Keywords-hard cutting materials; geometry angle; processing parameters; cutting tools

\section{INTRODUCTION}

With increasingly demanding equipments performing, the materials for them have become harder to cut [1]. The methods for cutting have become a hot topic in machining field. The main properties for high hard materials are highstrength, high-hardness, low thermal conductivity and hard to cutting. Selection and optimization of experimental parameters reasonably will make the best of tools in the cutting, which is one of the most effective solution [2,3]. However, through a large number of literature search it were found that with inadequate reference data from the related area, researchers often do their work from scratch, which have greatly increased their workload to find their results. The paper counted the processing parameters from about three hundreds related studying achievement, and the data analysis were done also.

\section{The Condition of Cutting Tools Applied}

For the high hardness materials, the main cutting tools include cemented carbides, ceramic and PCBN. In the Figure 1 , we can know that the most widely used is ceramic tools, which accounted for forty two percent of all tools. The cemented carbides and PCBN are thirty one percent and twenty seven percent separately. The condition above is drastically different from common metals cutting. The main reason for this is that ceramic material is more harder than cemented carbide, which cutting hardness materials better. Though the PCBN is harder than others, but their less toughness limited their application.

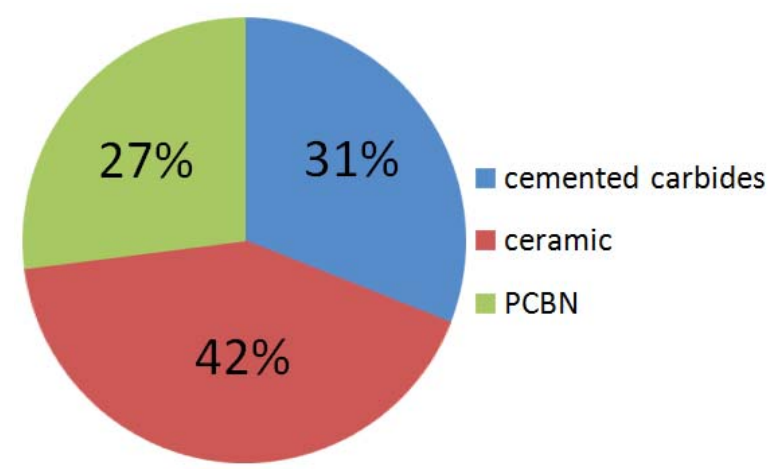

Figure 1. The percentage for different cutting tools

\section{The Condition of Rake Angle Applied for High} Hardness Materials CUTTING

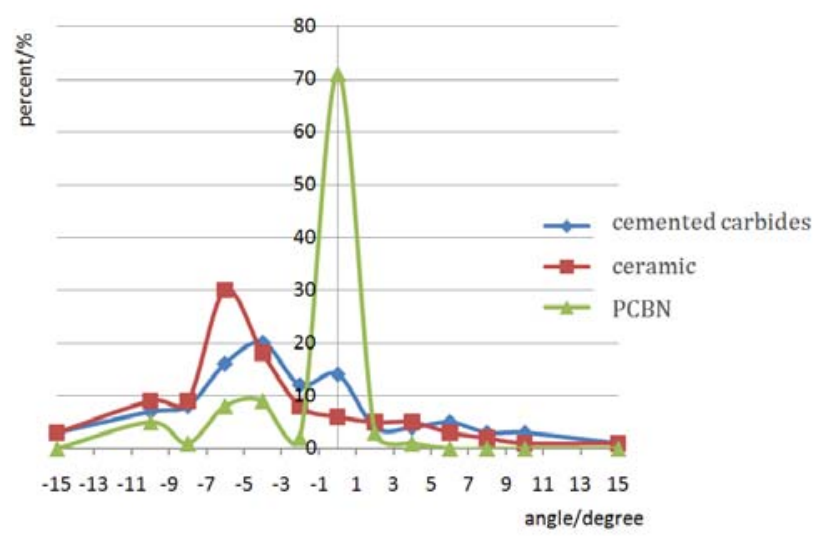

Figure 2. The best rake angle chosen for three kind of cutting tools

The rake angle is one of the most important parameter to the sharpness of cutting tools, whose choice will greatly affect tools life. In the Figure 2, it is noted that zero was chosen by seventy percent of PCBN. It is seem that the zero is the incontrovertible best choice. But with the given zero rake angle and 0.8 millimeters thick on PCBN material which limited rake angle choosing by grinding, the condition can be explained that the little optimization effort were done for PCBN materials. Besides zero degree, the best rake angle focus on range -5 to -7 , which is all of the noteworthy range. If we put together all of the negative rake, it is easy to find that sixty six percent of cemented carbides and seventy seven 
percent of ceramic take it for their choice, which are consistent with their toughness. In other words, in order to resist impact from the cutting process, the ceramic tools that possess less toughness than cemented carbides take more negative rake.

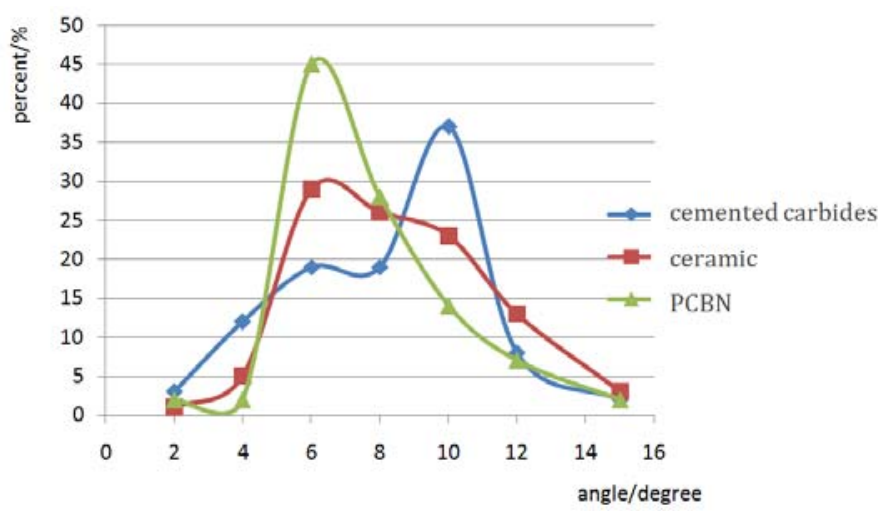

Figure 3. The best the relief angle chosen for three kind of cutting tools

The main function of relief angle is to reduce friction between flank surface of cutting tool and workpiece. In the Figure 3, it can be known that the flank angle chosen focus on range 4 to 12 degrees, which is similar with common choice range 6 to 8 . And the choices also conform to the law of normal distribution. When compared with the distribution of the middle line, it can be find that the applications for cemented carbides, ceramic tools and PCBN tools distribute separately according to ten degree, eight degree and six degree. It can be inferred that the PCBN tools need adopt small flank angle to improve impact resistance of tools. With the better toughness than PCBN tools, the tools by cemented carbides and ceramic can adopt bigger degree to make tools sharper.

When we look at the dates distributing on the edge of curve, it can be find that fifteen percent of cemented carbides, six percent of ceramic tools and four percent of PCBN choose their angle under four degree. At the same time, the angle for cemented carbides; ceramic tools and PCBN chosen more than twelve are ten percent, sixteen percent and nine percent separately. The condition shows that with the better toughness, the cemented carbides and ceramic can choose their relief angle in wide range. To the PCBN, the poor toughness limited their choice, which too big or too small angle chosen will make their application worse.

\section{[m The Condition of Tool Cutting Edge Angle Applied For High Hardness Materials Cutting}

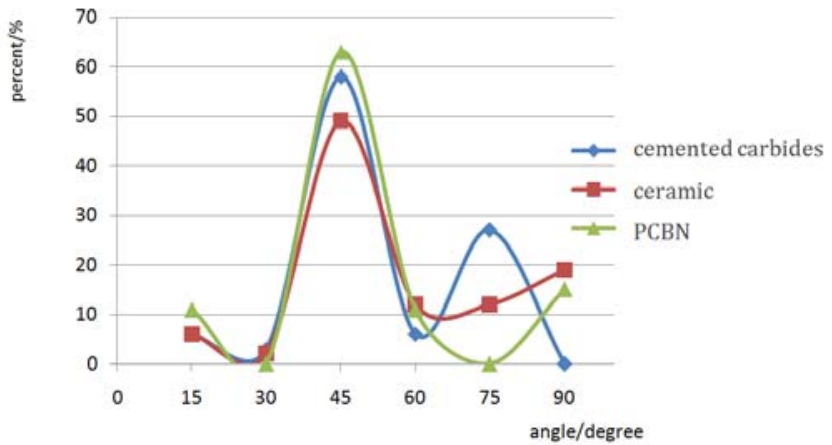

Figure 4. The best tool cutting edge angle chosen for three kind of cutting tools

From the Figure 4, it can be known that the most tool cutting edge angle used focus on 45 degrees. It shows that the 45 degrees is the optimal parameter for all three kinds of tools, which give an important parameter for the hardness materials cutting. Except 45 degree, another peak exist for the cemented carbides, which is 75 degree. But the scale for it is at relatively small number. For the ceramic tools, the 75-degree is about 12 percent, which is significantly less than 27 percent of cement carbides. The main reason for it is relatively poorly protection by the 75 degree, which limited the range of choice by ceramic tools with poor toughness. For the same reason, the PCBN cannot adopt this parameter. In general, the tool cutting edge angle given with 90 degree, in most condition, is to meet the demands of the shape of workpiece. The aim is irrelevant to hardness materials cutting, which will not discuss in this paper.

\section{↔ The Condition of Inclination Angle Applied For High Hardness Materials Cutting}

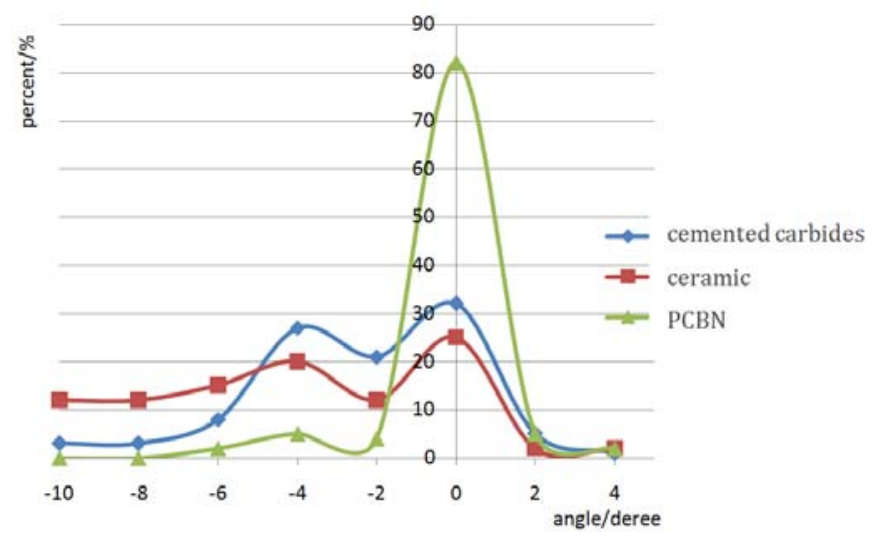

Figure 5. The best inclination angle chosen for three kind of cutting tools

The function of inclination angle is similar with tool cutting edge angle, which can improve the impact resistance of the cutter. The inclination angle chosen in the Figure 5 is similar to rake angles chosen in Figure 2, which main focus on 0 degree. It shows that it still little work, so 
far, have been done by the researchers. For the cemented carbides, the most inclination angle were chosen between minus 4 to zero. For the ceramic tools, the choice is between minus 10 to zero. When compared with choice under zero, the cemented carbides and ceramic tools are sixty two percent and seventy one percent separately. By comparison, the ceramic tools tend to choose more minus degree and more negative. It can be deduced that the negative degree is beneficial to the tools with poor toughness, which is a good choice for the hardness materials cutting.

\section{ACKNOWLEDGMENT}

The research supported by National Natural Science Foundation of China (Project number: 51275527 and 51475474).

\section{CONCLUSION}

1) For the hardness materials cutting, the best tools materials is ceramic, which is very different to the condition of metals materials cutting.

2) With the good toughness, the cemented carbides can choose their tool cutting edge angle, rake angle and inclination angle in more wide range. The tooling angles choosing by ceramic tools and PCBN tools with poor toughness within a small range.

3) Most tool cutting edge angle used focus on 45 degrees, which is the optimal parameter for all three kind of tools. It is a important parameter for the hardness materials cutting. The 75-degree is another choice for the cemented carbides tools, but its application frequency is less than 45 degree.

4) Zero rake angle and inclination angle was chosen for most of PCBN, which shows that the little optimization effort were done for PCBN materials.

5) The negative inclination angle has obvious effects against the tool breakage, which is a focal point for the hardness materials cutting.

\section{REFERENCES}

[1] X.Y. Zou, "The property and application prospect of hard cutting materials," heat treatment of metals journal, vol. 28, pp. 44-47, April 2003.

[2] Z.H. Long, X.B. Wang, and Z.B. Liu, "The rake face of cemented carbides for high speed cutting hardness materials," Journal of Tribology, vol. 25, pp. 83-87, January 2005.

[3] B.A. Zhao, "The turning for chilled steel," vol. 13, pp. 245 246, June 2003. 